\title{
Urgences
}

\section{À la mesure de l'espace}

\section{Helen Escobedo}

Numéro 17-18, octobre 1987

L'esprit des lieux

URI : https://id.erudit.org/iderudit/025427ar

DOI : https://doi.org/10.7202/025427ar

Aller au sommaire du numéro

Éditeur(s)

Urgences

ISSN

0226-9554 (imprimé)

1927-3924 (numérique)

Découvrir la revue

Citer ce document

Escobedo, H. (1987). À la mesure de l'espace. Urgences, (17-18), 131-139.

https://doi.org/10.7202/025427ar

Ce document est protégé par la loi sur le droit d'auteur. L'utilisation des services d'Érudit (y compris la reproduction) est assujettie à sa politique d'utilisation que vous pouvez consulter en ligne.

https://apropos.erudit.org/fr/usagers/politique-dutilisation/
Cet article est diffusé et préservé par Érudit.

Érudit est un consortium interuniversitaire sans but lucratif composé de l’Université de Montréal, l'Université Laval et l'Université du Québec à Montréal. Il a pour mission la promotion et la valorisation de la recherche. https://www.erudit.org/fr/ 


\section{Helen Escobedo \\ À LA MESURE DE L'ESPACE}

Depuis hier, les mots des poètes, des philosophes, des historiens d'art et des géographes m'ont envahie. Huit fois j'ai voulu courir à ma chambre d'hôtel pour changer le format de ma présentation d'aujourd'hui. Parce que je suis comme un caméléon: je prends la couleur de vos voix, je vous écoute et je voudrais illustrer vos esprits.

Mon inspiration, c'est, si vous voulez, les fréquences de lumière entre l'espace et la matière. Cela me fascine. Comme me fascinent les arbres dans la forêt et les échafauds autour des édifices, couloirs de colonnes qu'on voit tellement chez moi, en Grèce ou dans les diapositives qu'on vient de voir, là où le vide finit par les unir. J'essaie d'interrompre la lumière, d'employer l'espace en tant que matière scintillante et non en tant que matière solide. Voilà le sujet de mon inspiration et le but de mon travail.

Je pense que chaque lieu a son propre esprit, un esprit qui lui appartient tout particulièrement. Aucun lieu ne ressemble à un autre comme aucun homme, aucune femme ne ressemble à un(e) autre. Chaque homme, chaque femme comprend son lieu d'une manière différente. Il y en a même qui ne comprennent rien de manière différente. En tant que sculpteur, je m'inquiète de l'oeuvre que je crée afin qu'elle s'intègre le mieux possible au lieu que j'ai choisi ou qui a été choisi pour moi. Le lieu, alors, me parle. Je lui pose mille questions. Les gens du lieu me parlent. Je leur pose mille questions. Je m'impose, mais pas en lignes franches. Je m'impose doucement.

Je voudrais être, à partir de la lumière, de son feu propre, une extension du lieu. Je ne veux pas envahir l'espace mais le rendre plus remarquable. L'espace change continuellement et j'aide un peu cette métamorphose. Non pour m'imposer mais pour mieux le découvrir et, ainsi, changer ma façon de le percevoir.

Je ne suis pas artiste d'atelier, sauf quand je dessine. Et quand je dessine, c'est pour me découvrir un peu mieux. Je dessine tout le temps; parfois j'écris sur les dessins pour mieux décrire ce que je cherche, ce que je veux. C'est là que je trouve une grande liberté et aussi une grande richesse, car je garde ces dessins et, lorsque je les retrouve, ils me rapellent des images perdues dans mon esprit, d'autres idées, d'autres époques... Par eux je sais que, depuis toujours, la mesure de l'espace et la lumière me préoccupent. 
Voici une série de diapositives de mes oeuvres éphémères et de mes oeuvres permanentes, entremêlées de quelques autres qui ne sont pas de moi. Ces dernières sont des images qui m'entourent, des images dans mon jardin, dans mon pays et dans des pays que je connais bien, des images que je vole avec ma caméra car elles me parlent et font partie de cette collection de mémoires et de souvenirs que je cache dans mon âme pour que je puisse les sucer longtemps.

\section{COMMENTAIRES}

Je dessine pour moi, c'est vrai. Par le dessin, je commence à placer l'oeuvre dans son lieu, puis je change le lieu. Par le dessin, je peux vraiment savoir ce que l'espace donne dans différentes lumières ou encore me rappeler comment j'aurais voulu changer l'échelle, les couleurs, les matériaux du lieu.

Voici un monument très connu, à Mexico: le monument aux enfants héroïques tombés durant la guerre. Devant ce monument, il y a une sculture intégrée, qui est de moi. Mais comme c'est un trucage photographique, elle n'existe que sur cette photographié.

Parfois, je fais des choses comme ça, en plaçant mes maquettes là où il est possible d'établir une sorte de relation. Cette maquette-ci a deux mètres, elle n'est donc pas tellement grande: je l'ai mise là, un soir, quatre minutes exactement, pour prendre cette photograghie.

La maquette suivante a été faite pour le campus de l'Université de Mexico (celle qui est au nord de la ville). Elle a treize mètre de hauteur et s'intitule Falling Fence. Elle devait être en acier: ainsi elle aurait coûté beaucoup moins cher, mais on a décidé de la construire en béton et on a dû la refaire deux fois!

Voici la façon dont on plante les vignes, en Allemagne. Je trouve cela splendide. J'aurais voulu faire moi-même cette installation, mais j'aurais certainement ajouté des couleurs.

Voici maintenant la maquette de Falling Fence telle que je la voulais originellement. c'est une maquette de cinquante centimètres, en alumimium, mais cette fois vous la voyez placée sur le toit d'une voiture! On peut ainsi voir l'effet qu'elle aurait donné si on avait fait ce que j'aurais voulu: placée dans un grand étang, on aurait vu la maquette s'inscrire doublement, comme en un miroir. Ainsi, dans cette diapositive, l'image est rendue telle que je l'avais imaginée. 
Voici les toits de la maison que j'ai bâtie, en 1970, avec une équipe de travailleurs. Architecture sans architectes est un livre que j'ai toujours près de moi. Je pense que tout artiste devrait bâtir sa propre maison; c'est comme travailler la sculpture en fonction de ses besoins.

Signals: l'oeuvre est en Nouvelle-Zélande. Elle a quinze mètres de hauteur et elle est située à Auckland, tout près de la baie. Je voulais absolument qu'on puisse voir au travers la mer, les bateaux et tout ce qui bouge en bas. Quatre immenses échelles en aluminium peint sont placées l'une derrière l'autre et colorées en grosses bandes.

Voici une oeuvre éphémère, de trois étages de hauteur, que j'ai réalisée dans une galerie aux Etats-Unis: j' ai tout simplement suspendu, en spirale jusqu'au toit, quarante cadres de bois rouges.

Cette oeuvre est à l'Université de Mexico. Elle a quinze mètres de hauteur et elle est en acier peint dans mes couleurs favorites. C'est une oeuvre permanente qui s'intitule Coatl, ce qui veut dire serpent. Elle constitue une sorte de tunnel où les enfants prennent beaucoup de plaisir à jouer.

Voici une oeuvre éphémère, proposée au Contemporary Art Center, en Nouvelle-Orléans. Il s'agissait d'abord d'une fabrique, d'un espace alternatif où tout était vide. Lin Emery, une amie sculpteur, et moi, nous avons changé l'espace en faisant vingt choses différentes. Par exemple, nous avons pris les fenêtres et nous les avons refaites en carton et en bois. Elles sont suspendues au toit et totalement fausses. Derrière les radiateurs, nous avons placé des tubes de néon. Les colonnes sont enveloppées de tissu blanc et elles bougent car nous avons installé des ventilateurs électriques, bien cachés tout au haut. La passerelle, le long du mur extérieur, est illuminée par dessous et renvoie des étincelles, à travers les fenêtres.

Au Musée d'Art Contemporain, à Mexico, j'avais choisi les jardins, à l'extérieur, pour faire une installation éphémère. Le foin était le seul matériau disponible qui ne coûtait pas trop cher et qu'on trouvait tout près. J'avais demandé mille bottes de foin mais le directeur n'a pu m'en obtenir que cinq cents. L'oeuvre s'intitule Harvest Garden (1986). Le soir de l'inauguration, nous avons bien mouillé le foin à cause des fumeurs de cigarettes et nous avons fait une performance avec deux danseuses gymnastes.

Voici un couloir que j'ai fait pour que les enfants laissés là par leurs parènts puissent courir joyeusement au «kindergarden». C'est à 
l'école Montessori, à Mexico. Je me souviens d'une grande porte noire qui m'a engloutie lorsqu'on m'a envoyée à mon premier jardin d'enfants; j'ai passé la semaine à vomir chaque fois qu'on fermait la porte. Je n'ai pas voulu répéter mon histoire avec ces enfants. Là , ils peuvent jouer à cache-cache, aux indices, à la patinette...

Voici un bureau d'avocats. Le bureau n'a pas de fenêtres ou plutôt les fenêtres donnent sur d'autres bâtiments. C'est terrible. J'ai donc pensé qu'il serait gentil d'installer la forêt à l'intérieur. Alors, j'ai placé plus de cinq cents poutres en bois, un peu au hasard, le long des corridors.

Deux architectes et moi avons travaillé en équipe et nous avons gagné un concours pour construire ce bâtiment en béton coulé. Nous avons très bien travaillé ensemble et nous avons pris sans problèmes, à nous trois, les décisions qu'il fallait prendre. Je pense que les artistes devraient travailler plus souvent en équipe et avec d'autres professionnels.

Voici une oeuvre éphémère qui a duré trois semaines et que j'ai réalisée avec quinze étudiants de l'école d'architecture, à l'Université de Tulane, en Nouvelle-Orléans. L'oeuvre a cent mètres de longueur. Il s'agit de tubes de carton peint que la Container Corporation of America m'avait donnés.

A Mexico: voici une proposition pour un monument aux pompiers. Je n'ai pas pu le réaliser parce que le $1 \%$ a été réduit à $0 \% .$. Cette situation se présente souvent: on nous commande l'oeuvre, on travaille, on réalise la maquette et, finalement, il n'y a pas d'argent.

Voici la maquette d'une oeuvre qui s'intitule Homage to Stonehenge. Cela continue d'être un hommage, et cela continue aussi d'être une maquette... La compagnie qui l'avait commandée a fait faillite trois semaines après avoir signé le contrat pour cette sculpture qui devait avoir 25 mètres de longueur. C'est triste de beaucoup travailler à des dessins, à des maquettes pour une oeuvre de commande qui, parce qu'elle est permanente, coûte forcément très cher; et il arrive tellement souvent que l'argent fasse finalement défaut. Ainsi, pour pouvoir travailler constamment et voir des résultats immédiats, je me réfugie dans mes oeuvres éphémères, conçues à partir de n'importe quels matériaux.

Curieusement, des images reviennent parfois et on se demande: «Est-ce que je les ai déjà vues? Est-ce que j’ai déjà vécu cette image-ci? Est-ce que je l'ai déjà avalée sans m'en rendre compte? Ou 
encore est-ce que je choisis continuellement des lieux et des objets qui ressemblent à mon oeuvre?" Par exemple, cette photographie, prise en Allemagne: là, ils utilisent la broche à poule pour maintenir sur les collines la terre et les pierres afin d'éviter qu'elles tombent le long des autoroutes. J'utilise aussi souvent ce matériau parce qu'il est excellent pour mes installations transparentes.

Voici maintenant le matériel que vous avez vu sur la montagne et les diverses utilisations qu'on peut en faire. Par exemple: ajouter des branches aux arbres, tout doucement, ou encore intervenir dans un espace que j'ai vu en hiver pour y faire revenir l'automne. lci, ce sont des cylindres dans des cylindres dans des cylindres: jaunes, oranges, rouges. Ce n'est pas une photographie truquée, j'en ai fait soixante exemplaires. Lorsqu'il n'y a pas de soleil, les cylindres sont très présents, très solides. Ce jour-là, il faisait très beau; alors, le soleil perce les cylindres et ils deviennent des fantômes colorés.

Nous sommes maintenant à Jérusalem, en 1986. Je suis restée là trois semaines sans savoir vraiment quoi faire. La ville, entièrement recouverte de pierres jaunes, est très belle. C'est une ville dorée. Je voulais attraper la lumière et je ne savais pas comment m'y prendre. Voici comment on attrape la lumière: The Great Cone (1986). Et comment on retient l'eau, car c'est justement là que l'oasis commence et que le désert se termine. L'oeuvre est en perpétuel changement: à midi, elle se présente comme un dessin au crayon et la couleur disparaît totalement. L'été, les couleurs en sont très fortes. Au printemps, il fait très froid et tout devient vert. Ce n'est pas que la sculpture change réellement, c'est qu'elle est transparente mais solide. Le grand cylindre extérieur, fait de barreaux d'acier carrés, mesure huit mètres de hauteur par sept mètres de diamètre. Dedans, fixé au cylindre de la partie supérieure, vous voyez un grand cône, fait aussi en carrés d'acier mais peint en jaune vif. Quand le soleil illumine le cône, il change de couleur, il devient mi-jaune, mi-orange; parfois c'est le cylindre qui disparaît presque entièrement ou vice versa. C'est une oeuvre qui me plaît beaucoup mais, plus encore, elle plaît aux gens qui habitent là.

Voici une sculpture transportable. Règle générale, c'est moi qui me transporte et, alors, je fais les choses sur place. Ici, ce sont mes "nuages» transportables. Ce sont des espèces de stores vénitiens qu'on peut rouler, mettre dans des tubes et emporter en voyage. La hauteur, le placement et, surtout, ce qui est en bas changent selon les lieux d'exposition. Quand je les ai présentés à Berlin, j'ai mis un énorme cercle rouge: c'était le mur de Berlin. Ici, ce sont des nuages orageux sur un Mexique plein de brouillard: j'ai installé des sections 
de cylindres de carton peints en noir gris et blanc, comme des cheminées, sous les nuages.

Voici une installation que j'ai faite, il y a trois semaines, alors que j'étais dans un congrès de femmes sculpteurs. On attendait cinq cents femmes mais plus de mille sont venues. J'ai fait une intervention dans un parc public. Nous avions très peu d'argent. Il fallait agir un peu comme ici et utiliser les matériaux qui étaient déjà sur place. Alors, avec des branches, j'ai commencé à faire des balais. Les femmes et les balais, de beaux balais pour de belles femmes, dans mes couleurs favorites: le jaune, l'orange et le rouge. Chez vous, on n'utilise plus de balai, tout est mécanique. Chez nous, on fait encore les balais à la main: un petit homme passe chaque mois et vous vend le matériel nécessaire. Cette oeuvre s'intitule, titre intraduisible, Sweeping Statement! Chaque balai a cinq mètres de hauteur et il a fallu trois personnes pour les placer contre les arbres. Ils sont très lourds.

Je vous parlerai maintenant de celle de mes oeuvres que je considère comme l'une des plus belles. J'ai fait partie d'une équipe de six sculpteurs invités par le recteur de l'Université nationale autonome de Mexico; les artistes travaillaient déjà à l'Université comme professeurs, techniciens, ou encore, dans mon cas, comme directrice de musée et de galerie. Un jour, le recteur nous a donc invités sur l'emplacement du futur centre culturel universitaire. On allait bâtir une salle de concert, une bibliothèque, des théâtres: ce serait, en quelque sorte, le petit Lincoln Center de Mexico-Sud. Avec un geste généreux vers l'horizon, le recteur a dit: "faites-moi quelque chose". Nous nous sommes regardés tous les six. Nous étions vraiment de trois générations: le plus connu, est un sculpteur de soixante-dix ans, Mathias Goeritz, (il a fait les fameuses tours de la cité-satellite avec Luis Barragan, le grand architecte), et le plus jeune a trente-huit ans. Nous nous sommes donc regardés en pensant que, finalement, nous devions faire chacun une oeuvre. Mais quand nous avons retrouvé le geste du recteur, nous nous sommes dit: "pourquoi ne pas faire une seule oeuvre, sans la signer». Et nous avons réalisé ce qui est connu désormais comme l'Espacio Escúltorio (1978). À l'intérieur, on voit aujourd'hui la lave que les géologues nous disaient être dessous et c'est pourquoi nous avons enlevé absolument tous les cactus et tous les arbres. Voyez l'immensité de ce lieu. Je voulais monter et descendre en hélicoptère pour prendre des photographies mais on ne m'en n'a pas donné la permission. Nous avons fait ici des performances: il est facile d'y asseoir trois mille personnes! J'y ai vu des danseurs, des acteurs; on y donne aussi des concerts et l'acoustique est extraordinaire. Voilà vraiment l'esprit d'un lieu. Et l'esprit de ce lieu je l'explique ainsi, simplement: le premier cercle est un hommage à la 
géologie du site, le deuxième cercle (soixante-quatre pyramides ou triangles isocèles) est un hommage à l'homme, et le troisième, placé autour des deux autres, préserve, à cent mètres, la verdure et tout ce qui appartient au site comme les arbres et les cactus. C'est un lieu très magique, assez mystique. Personne ne peut vraiment le décrire, le photographier, le filmer; il faut le voir, le sentir, le vivre. Pour ce faire: vous entrez par l'une des quatre portes principales (nord, sud, est, ouest) et, quand vous êtes au centre, vous vous retournez et vous élevez vos yeux vers le ciel qui devient une grande coupole.

C'est un esprit que je voudrais connaître à nouveau. Encore une fois dans ma vie, je voudrais pouvoir travailler comme cela, dans l'anonymat, peut-être avec un groupe d'artistes. Pas toujours avec le même groupe, car c'est impossible. De plus, l'expérience n'a pas du tout été facile. Mais quand je vois ce que nous avons réussi à réaliser cette fois-là, j'aurais envie de recommencer ailleurs, avec d'autres copains, peut-être dans d'autres pays, avec d'autres lumières et d'autres réalités.

\section{Espace - Place}

Genus Loci - Esprit du lieu

Tout cela adhère à mon identité écologique, une identité qui oscille entre le comportement poétique et la vie quotidienne, entre ma vision et ma réalité. J'interviens entre la nature et l'homme d'une façon aléatoire, les nouant tous deux ensemble comme en un tissu harmonieux et, ce faisant, je trouve ma liberté. Liberté de penser, d'agir, de créer pour tel lieu, à tel instant, dans tel espace. 


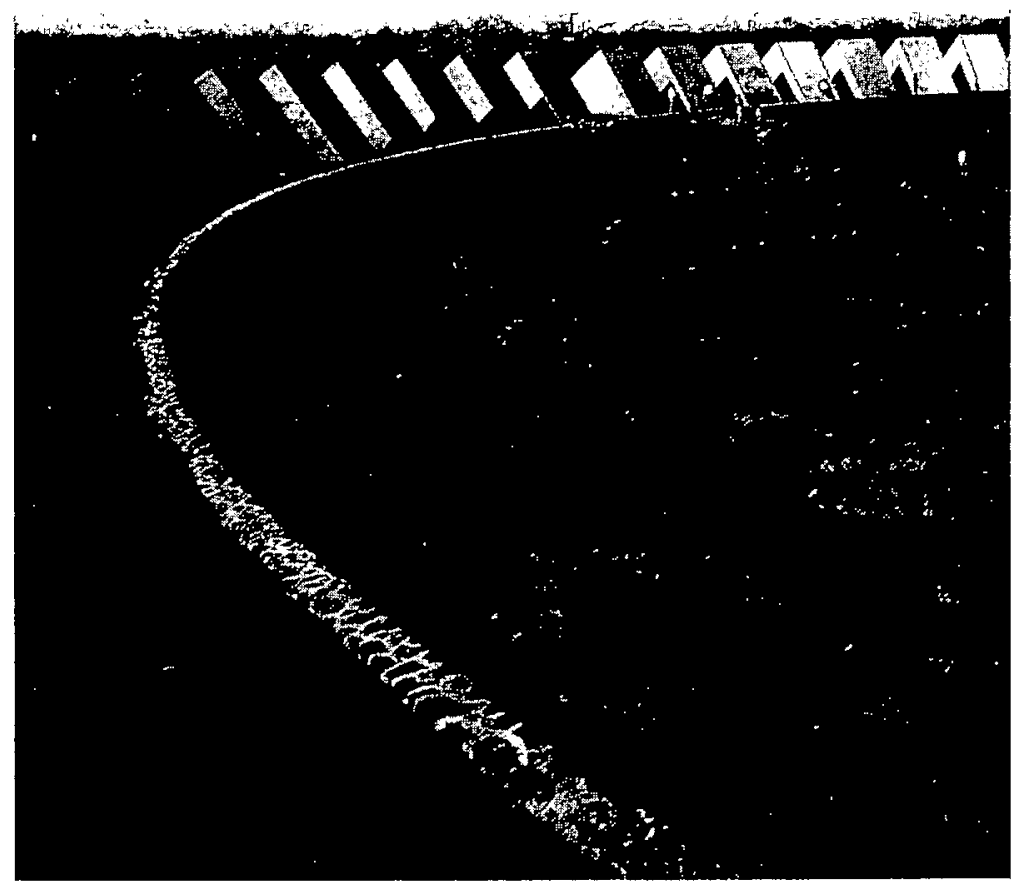

El espacio escultórico (diamètre: $120 \mathrm{~m}$ )

Création de Helen Escobedo, Manuel Felguérez, Mathias Goeritz, Hersúa, Sebastián et Féderico Silva.

Universidad Autónoma de México. 


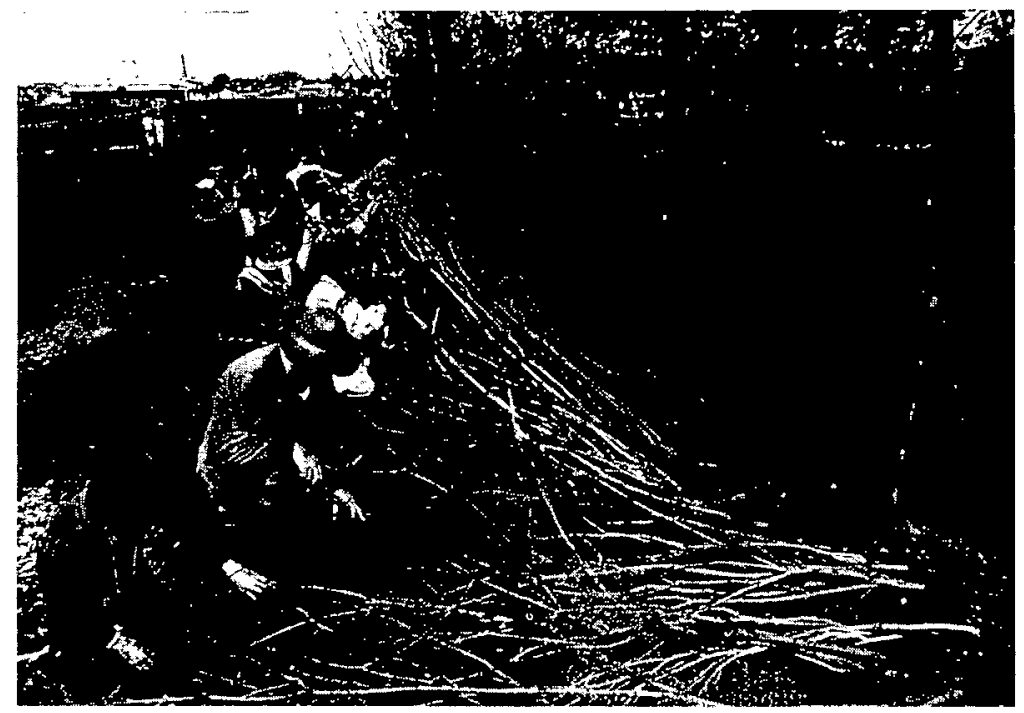

Installation conçue par Helen Escobedo, réalisée au Parc Beauséjour de Rimouski (juin 1987)

L'équipe de travail

(photo: Gérald Bouillon)

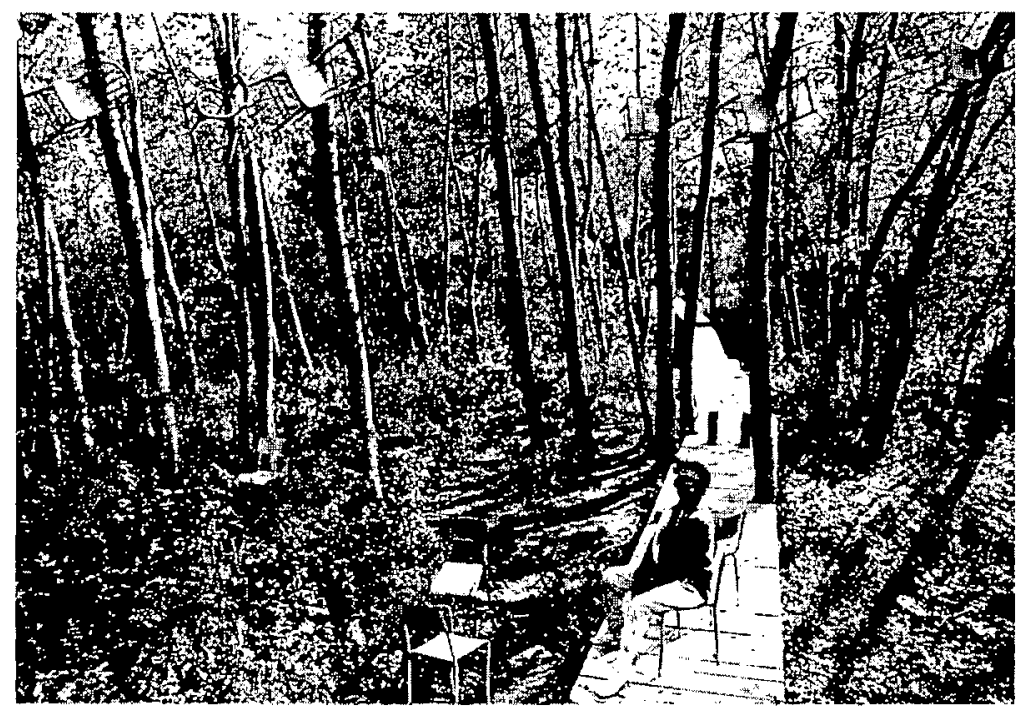

Installation conçue par Helen Escobedo (juin 1987)

Une partie de l'oeuvre réalisée au Parc Beauséjour... et son auteure.

(photo: Gérald Bouillon) 